\title{
LA MATEMÁTICA COMO HERRAMIENTA EN LA CONSTRUCCIÓN Y CONOCIMIENTO DEL ENTORNO
}

\author{
JOHN FREDDY RAMIREZ CASALLAS
}

\section{LA CAPACIDAD NARRATIVA}

Todos nuestros alumnos pertenecen a entornos particulares que son bastante complejos. Están compuestos por elementos culturales, sociales, históricos, físicos, etc. Estos entornos se ven transformados cuando los estudiantes migran o cuando sin migrar se presentan cambios geográficos, culturales, sociales. En consecuencia se debe aceptar que los entornos evolucionan. Esta evolución se da porque a su interior se dan transformaciones de los elementos que las componen, como de las relaciones que entre éstos y con otros entornos se presenten. Tales cambios pueden ser provocados por factores internos o externos. Es el caso del fenómeno del Niño que ha provocado, en nuestras localidades, transformaciones hidrológicas (disminución o aumento de los cauces de agua), sociales (formas de vestir, periodos de veraneo), etc.

Entre los agentes que pueden provocar cambios del entorno se encuentra el hombre. Es un componente indispensable del entorno como lo hemos definido y podemos aceptar que ha sido uno de los agentes que (para bien o para mal) ha provocado enormes cambios en el mismo. Es imprescindi-

1. Licenciado en Matemáticas y Física de la Universidad del Tolima. Maestro temporal del Instituto Microempresarial El Totumo, lbagué. ble porque sin el hombre no existirían entornos culturales y viceversa. Es uno de los principales agentes de cambio hasta el punto que ha podido transformar enormes selvas en ciudades, escudriñar el universo para llegar a Marte. Cambios, que por cierto, hoy se busca sean lo menos dañinos posible.

Todos nosotros (estudiantes, padres de familia, profesores) pertenecemos a uno o varios entornos. Pero el sólo hecho de pertenecer a él (ellos) no nos garantiza que lo(s) conozcamos. Es el caso de nuestros estudiantes, no basta con existir físicamente en el entorno para comprender la realidad que vive. Tanto ellos como nosotros debemos construir y comprender la realidad. Para hacerlo debemos desarrollar nuestra capacidad de narrar el mundo, el entorno, a través del lenguaje. Es mediante el lenguaje que construimos y comprendemos la realidad. A la capacidad de narrar la realidad, leerla, le llamaremos capacidad narrativa.

En síntesis, podemos plantear que debemos comprender y construir la realidad, para ello necesariamente debemos desarrollar nuestra capacidad de narrarnos. Estos procesos se encuentran estrechamente ligados: desarrollar una nueva construcción del entorno hace posible una nueva comprensión de él y viceversa.

Veamos mediante algunos ejemplos lo que lo anterior significa. Nuestros estudian- 
tes pueden habitar entornos que se caracterizan por altos índices de desempleo. El solo hecho de habitar allí no les garantiza que comprendan y posean una construcción propia de esa realidad. Es tan así que puede haber estudiantes que ni siquiera sepan que existe el problema. Otros pueden saber que existe, pero no comprender las múltiples causas que lo provocan.

Otro ejemplo que muestra la validez de las afirmaciones hechas, pertenece a actividades en clase de matemáticas que desarrollamos con estudiantes de grado sexto ${ }^{2}$. De común acuerdo nos propusimos investigar un problema del entorno al que pertenecen. Según algunos de ellos, en varias familias hay niños que no asisten a la institución escolar porque no tienen dinero para pagar sus estudios. Intentando saber que tan extenso y regular es el problema ${ }^{3}$, los estudiantes encuentran familias de sus veredas. Los datos recogidos, sin permitirnos dar una última respuesta al problema planteado, los aprovechamos para trabajar nociones de estadística y relaciones de orden entre números naturales. Reuniendo los grupos de acuerdo a sus respectivas veredas, respondimos preguntas como: ¿Cuántas personas estudian? ¿cuántas personas no estudian? ¿Es mayor el número de personas que estudia del que no estudia?

Para finalizar la actividad los estudiantes escribieron un cuento con base en el estudio que hicieron. De todos los trabajos se extractaron dos conclusiones muy importantes: los estudiantes se concientizaron de que hay estudiantes que no estudian por falta de dinero y otros porque no quieren hacerlo. Por otro lado, se sorprendieron bastante con el hecho de haber encontrado una vereda muy diferente a la que siempre habían pensado, una con gente amable y con problemas, con parajes feos y bonitos. Todo ésto muestra que los estudiantes conocieron y reconstruyeron la imagen que tenían de su entorno.

\section{JUSTIFICACIÓN DE HIPÓTESIS. OPCIONES DESDE LA ESCUELA}

Hasta el momento hemos mostrado que es posible conocer y construir el entorno, y que es necesario hacerlo mediante la narración del mundo, lo que implica usar el lenguaje. Pero ¿qué pasaría si el conocimiento que manejamos en la Escuela no permite que los estudiantes comprendan y construyan su entorno? ¿Qué importancia tiene para los estudiantes la construcción y comprensión del entorno?

Para dar respuesta a estos interrogantes, partiremos de una tesis que actualmente estamos defendiendo ${ }^{4}$. Existen conocimientos escolares y formas de enseñanza que no permiten la construcción y comprensión del entorno. Estos conocimientos pueden presentar contenidos sobre el entorno, pero estar desligados de las construcciones que los estudiantes han desarrollado alrededor de él. Teniendo en cuenta que tales construcciones pueden desarrollarse por fuera de la Escuela, dentro de ésta se puede llegar a privilegiar conocimientos irrelevantes como generadores de conexiones con sus mundos propios que traen consecuencias tan grandes como: separación ge-

2. Los estudiantes pertenecen al Instituto Microempresarial El Totumo, ubicado en zona rural de Ibagué. Las actividades se realizaron finalizando el semestre A de 1997.

3. Desde la Institución se viene liderando, con otras instituciones del sector, un trabajo para conocer qué sucede en la región alrededor de este problema.

4. Véase John Freddy Ramírez. Acercamientos a un modelo democrático en la enseñanza de la Física (ver partes 2 y 3). Trabajo de grado presentado para optar el título de Licenciado en Matemáticas y Física. Semestre A de 1997. También puede consultarse versión abreviada. 


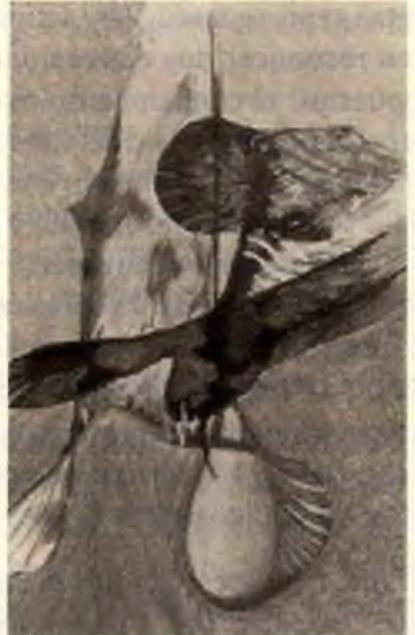

llustración: Claudia P. Losada

neral entre la realidad de su mundo propio y una realidad constituida poro conocimientos escolares; aletargamiento de la capacidad crítica sobre su mundo; decaimiento de la capacidad de reconstruir el mundo; aletargamiento intelectual. En ningún momento se niega que los estudiantes estén en contacto con el entorno, pero se resalta que así como las interacciones con el entorno pueden frenarse mediante el conocimiento escolar, también es posible enriquecerlas a través de él. De todo lo anterior se pueden extractar algunas de las consecuencias de no construir y comprender el entorno con nuestros estudiantes, e imaginar su importancia.

En lo que concierne a nuestro papel como maestros podemos preguntarnos: ¿qué podemos hacer desde la Escuela? Responderemos a esta pregunta con una propuesta: debemos retransformar el conocimiento escolar hacia uno que enriquezca las interacciones de los estudiantes con el entorno. Todo lo anterior implica que abramos las puertas a otras formas de ver el mundo, que en interacción con los estudiantes avancemos en el enriquecimiento de sus formas de ver el entorno. En ningún momento significa excluir todos los conocimientos que normalmente se han tratado en la Escuela, sino entender que estos conocimientos deben hacer posible que los estudiantes complementen, cuestionen sus lecturas sobre la realidad, reconstruyan y logren mejores comprensiones sobre sus entornos, sus mundos. Todo ésto implica entender que la institución escolar se desarrolla, como mínimo, con base en la intencionalidad de desarrollar tales interacciones. Llevar a cabo tal tarea exige desarrollar las capacidades linguiísticas de nuestros estudiantes, abrir espacios para que desarrollen su autonomía $y$, necesariamente, comprometernos en investigarnos e investigar nuestro mundo de vida.

\section{DLAGNóSTICO}

Para mostrar el sentido práctico de esta propuesta, es relevante exponer el diagnóstico particular que se ha hecho con nuestros estudiantes. Tal tarea puede ir antecedida de un pequeño marco teórico que nos permita responder preguntas como: ¿cuándo sabemos que se está desarrollando la capacidad narrativa en conexión con el entorno? ¿En qué formas podemos desarrollarla?

\section{Elementos teóricos sobre el lenguaje $\mathrm{e}^{5}$}

Cuando un individuo expresa algo siempre se pueden identificar dos planos: uno de expresión (mundo representante) y otro de contenido (mundo representado). Consideraremos que el primero está compuesto por significantes y el segundo por significados. El significante es ese "algo" que se relaciona con el significado y que nos sirve para expresarlo. Quien interpreta el signifi-

5. Los conceptos centrales aquí planteados (signo, código, significante, significado, etc.) son tomados de Tratado de Semiótica General de Umberto Eco. 
cado posee códigos que relacionan significantes y significado.

De lo anterior se deriva que individuos con códigos diferentes pueden asignar diferentes significados a un mismo significante Ésto se hace evidente en una clase: mientras el maestro maneja un código particular en el desarrollo de su clase, cada uno de los estudiantes posee códigos que les permiten interpretar lo que allí sucede. Si el código se identifica con el del maestro, entonces logrará comunicar al estudiante el mensaje que deseaba, pero si el código es diferente el mensaje se alejará de aquel que quería comunicar el maestro. Lo dicho supone que los estudiantes sí pueden interpretar lo que se comunica en clase, sin importar si construyen o no el mensaje deseado.

El conocimiento que es elaborado por los estudiantes se codifica en sistemas simbólicos ${ }^{6}$ variados y se puede discriminar en conceptual y de procedimiento. El primero se basa en elaboraciones semánticas (manejando el contenido de las expresiones) y el segundo en las sintácticas (manipulando las reglas que relacionan las expresiones simbólicas). Es el caso en donde se pide a un estudiante solucionar la ecuación $x+5=$ 4. Un estudiante que responda: se pasa 5 a restar al otro lado y obtenemos que x es - 1 , ha hecho una elaboración sintáctica. Otro que responda algo como: supongo que tengo cinco pesos $y$ hay una cantidad $x$ que al ser sumada con 5 hace que quede con 4 pesos. Esa cantidad quita un peso a la inicial, por tanto $\mathrm{x}$ es $\mathbf{- 1}$, donde el símbolo menos (-) significa que quita, lo que sería un caso de elaboración semántica.

\section{El conocimiento matemático}

Como todo conocimiento, el matemático hace uso de dos mundos: uno represen- tante y otro representado. Alrededor de él se pueden reconocer dos dimensiones polares y opuestas: el conocimiento matemático determinado desde la disciplina y otro determinado desde la interación con el entorno. A los mundos representante y representado los llamaremos A y B respectivamente, $y$ a los representantes y represeantado desde la interacción con el entorno los llamaremos $C$ y $D$ respectivamente. Entre los mundos representante y representado existen códigos. Los $\mathrm{C}_{\mathrm{AB}}$ son los códigos entre $\mathrm{A}$ y B, $C_{\mathrm{CD}}$ son los códigos entre $\mathrm{C}$ y D.

Cuando los códigos $\mathrm{C}_{\mathrm{AB}}$ que maneja un estudiante coinciden con los que propone la disciplina, entonces el conocimiento q̨ue resulta de relacionar A y B será considerado correcto, lo que significa decir que el estudiante ha tenido un buen aprendizaje. Cuando $\mathrm{C}_{\mathrm{CD}}$ son correctos desde la perspectiva de la disciplina, se dirá que la aplicación que el estudiante hace del conocimiento matemático en su entorno es correcta (Figura 1).
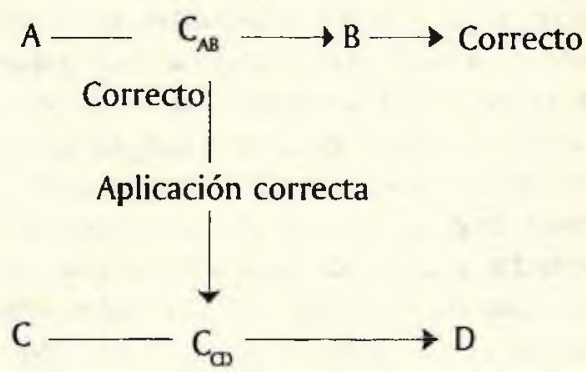

Correctos

Figura 1. Aplicación en el entorno del conocimiento matemático aprenhendido.

Pero resulta que también hay casos en donde $\operatorname{los} \mathrm{C}_{\mathrm{AB}}$ y los $\mathrm{C}_{\mathrm{CD}}$ pueden ser incorrectos desde la perspectiva de la disciplina. Cuando lo sean los $\mathrm{C}_{\mathrm{AB}}$ se dirá que el estudiante no ha aprenhendido correctamente el conocimiento planteado, y cuando lo sean

6. En el caso de las matemáticas tenemos símbolos muy conocidos como:,,$+-=$, , más, $\%$, o combinaciones de símbolos como: $\mathrm{a} \times \mathrm{b}=\mathrm{A}, 2 \mathrm{X}=2 \mathrm{Y}=0$, carros azules + carros rojos $=$ carros. 
los $C_{\infty}$ se dirá que el estudiante no lo aplica bien.

En síntesis, tanto $\mathrm{A}, \mathrm{B}, \mathrm{C}, \mathrm{D}, \mathrm{C}_{\mathrm{AB}}, \mathrm{C}_{\mathrm{CD}}$ son variables. Los $A, B$ son determinados desde la disciplina y dependen de la temática que se desea abordar. Los C, D dependen del entorno y se plantean en conexión con los A, B. Los $C_{A B}$ y $C_{C D}$ pueden ser o no correctos desde la perspectiva de la disciplina.

Estos códigos agrupan todos los que manejan los estudiantes. Por otra parte, es bien sabido que la disciplina matemática usa un lenguaje muy particular denominado matemático. En la medida que aumenta el grado de formalización las expresiones simbólicas se hacen más universales e identificables como del lenguaje matemático. Son esas expresiones las que se ubican en $\mathbf{A}$.

Consideremos que en el aprendizaje de las matemáticas acceder desde $\mathrm{A}$ a $\mathrm{C}$ es todo un proceso que involucra pasar por formas de representación intermedias. Lo anterior significa decir que no se pasa de $\mathrm{A}$ a $\mathrm{C}$ directamente sino que hay formas de representación que sirven de puentes. Igualmente sucede con los $C_{A B}$ y los $C_{C D}$. Existen códigos intermedios, como también existen mundos representados intermedios entre B y D. Así es posible pensar que un estudiante puede hacer acercamientos graduales entre el conocimiento matemático según la disciplina y el contextualizado en el entorno (Figura 2).

Todo lo anterior implica dos cosas fundamentales: la conexión del conocimiento matemático con el entorno y reconocer que los estudiantes poseen formas de representación propias que se deben conectar con las propuestas por la disciplina.

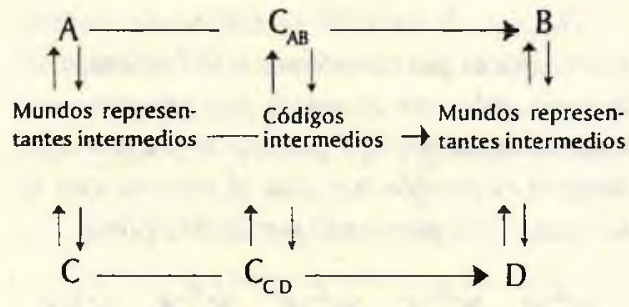

\section{Correctos}

Figura 2. Conexiones entre $A, B, C, D, C_{A B}$ y $C_{C D}$

La necesidad de conectarnos con el entorno ya fue desarrollada en la justificación de hipótesis. Exploremos, entonces, por qué es posible.

Partamos de considerar que vivimos un mundo físico que presenta las suficientes regularidades como para ponernos de acuerdo acerca de lo que sucede en el entorno. Es así que personas que se encuentran cerca pueden experimentar una lluvia y compartirla. Igualmente el conocimiento matemático, al menos como lo conocemos, es posible por esas regularidades. Par comprenderlo basta imaginar qué pasaría si la materia fuese demasiado inestable de manera tal que en un lapso de 10 segundos pudiese perder sus características iniciales. En un mundo así ir a un banco para pagar una cuota de 100.000 pesos como abono a la deuda de una casa sería un riesgo; en una fila de personas que demorase más de 10 segundos podía transformarse íntegra la plata que llevamos para pagar la cuota. Esa imposibilidad de saber como se transforman haría imposible, al menos para nosotros, aplicar y construir las operaciones aritméticas más simples que podrían servir para desarrollar los algoritmos que nos permitan calcular cuánto quedamos debiendo de la casa.

7. Aquí se hace referencia al aprendizaje del conocimiento donde la conexión con el entorno ha sido ampliamente corroborada. Es el caso de conocimientos que tratamos en nuestras escuelas, como el cálculo, álgebra, aritmética, trigonometría. Tal aclaración es necesaria si se tiene en cuenta que como disciplina la matemática construye nuevos conocimientos que pueden no tener conexión con el entorno. Es el caso de la topología que un buen tiempo fue apenas una teoría matemática que actualmente es bastante aplicada en física. 
De todo lo anterior se desprende que las matemáticas que conocemos están íntimamente articuladas con el mundo que experimentamos. De esta manera podremos aceptar que siempre es posible conectar el entorno con el conocimiento planteado por la disciplina ${ }^{7}$.
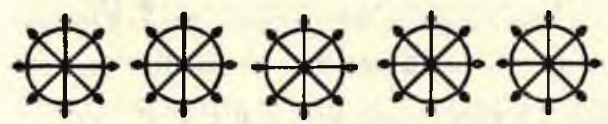

En lo que concierne a las formas de representación que posen los estudiantes es necesario conectarles con las propuestas por la disciplina porque de no hacerlo ellos estarían inhabilitados para desarrollar autónomamente nuevas conexiones del conocimiento con el entorno, lo que justifica su necesidad y es posible porque ninguna persona al convertirse en matemático ha tenido que renunciar a las formas de representación que antes usaba. Posibilidad que se refuerza por una segunda razón: biológicamente estamos preparados para aprender nuevos lenguajes $y$, de hecho, diversas formas de apreciar el mundo. De allí que desarrollar esa posibilidad dependa más de nuestra capacidad como maestro para facilitar desde la escuela ambientes culturales que enriquezcan y orienten el acercamineto al lenguaje matemático, sin desconocer que es diferente del lenguaje usado por nuestros estudiantes y de las formas intermedias que permiten llegar a él.

\section{3. ¿Se está desarrollando la capacidad narrativa?}

Después de todo lo anterior, ahora si podemos exponer los criterios que nos permitan determinar cuándo sabemos que a través del conocimiento se está desarrollando la capacidad narrativa en conexión con el entorno y, por lo tanto, haciendo de la matemática una herramienta en la construcción y conocimiento del entorno.

Entendemos que alrededor de una temática un estudiante ha desarrollado la capa- cidad narrativa en conexión con el entorno cuando por sí solo puede tomar el conocimiento que le ha planteado la disciplina y recrearlo correctamente en el mundo que lo rodea. Lo anterior implica que lo aprehendido haya aportado en una reconstrucción del mundo hecha por el individuo, que sus lecturas sobre la realidad la revelen. Es un individuo que ha hecho del conocimiento un medio para comprender de nuevas maneras su mundo, incluido él.

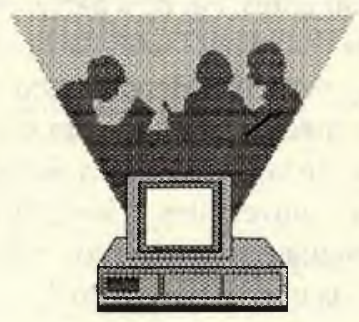

Todo lo anterior implicaría aceptar que el estudiante avanza gradualmente en la consecución de los $\mathrm{C}_{A B}$ y $\mathrm{C}_{\mathrm{CB}}$ correctos, como el conocimiento y manipulación de los elementos intermedios (mundo representante y representado, códigos intermedios). Tal gradualidad se acepta considerando que el esquema de la Figura 2 representa un sistema que se transforma, crece y enriquece orientándose hacia el planteado en la Figura 1. Además, lo dicho trae como consecuencia aceptar que desarrollar la capacidad narrativa no implica haber alcanzado los últimos grados de formalización pero si avanzar hacia ellos.

Los individuos que no están desarrollando esa capacidad narrativa ( $\mathrm{CN}$ ) en conexión con el entorno, a diferencia de los que si lo hacen, verían roto el esquema de la Figura 2. Por ejemplo, es posible que no usen formas intermedias de representación y solamente se concentren en trabajar de $A$ hacia $B$, ante lo cual las otras relaciones quedarían abandonadas.

Un ejemplo de fracciones nos permitirá entender lo anterior. Un estudiante que esté 
desarrollando la $\mathrm{CN}$ alrededor del entorno debería estar manejando códigos $\mathrm{C}_{\mathrm{AB}}$ cercanos a los correctos y, además, puede pasar por los elementos intermedios para aplicar ese conocimiento en el entorno, en problemas de su vida. Esto en ningún momento significa, apenas, aprender a solucionar problemas del tipo "si una parcela es $3 / 4$ de...", sino poder construir enunciados, problemas en conexión con su entorno. Lo anterior en ningún momento excluye que el estudiante pueda plantearse problemas que conciernen a la disciplina, por ejemplo: ¿qué otra fracción representa lo mismo que $3 / 4$ ? De hecho, el estudiante solucionará de manera muy particular los problemas, dependiendo de las características que en él tome el sistema representado en la figura 2 . Por lo cual el problema puede resolverse con elementos de la misma disciplina (ecuaciones de equivalencia) o con elementos combinados que involucran elementos de la disciplina y del entorno, como es el caso de tomar pedazos de papel con unidades iguales y diferentes divisiones y llegar a que $3 / 4=6 / 8$ corresponden a la misma cantidad.

A pesar que no se ha logrado la formalización de las fracciones, un estudiante que comprenda lo que sucede con los papelitos está muy cerca de plantear problemas con sentido articulados en su entorno. En este caso también se considera que se está desarrollando la $\mathrm{CN}$ en conexión con él.

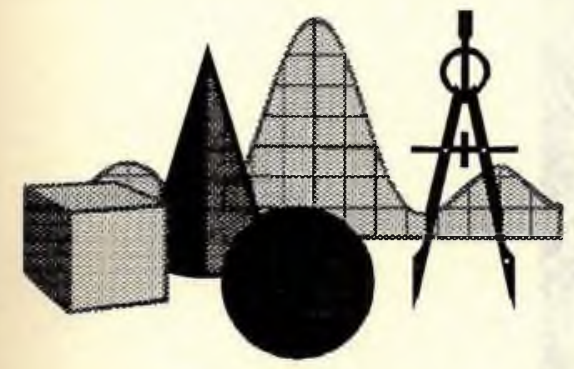

\section{Nuestros estudiantes}

En el Instituto Microempresarial El Totumo me corresponden cinco cursos (de sex- to a once, excepto octavo), uno por grado de seis que tiene la institución.

En los últimos cuarenta y cinco días del semestre A del presente año (primeros días de mi trabajo en la institución) se realizaron clases y, simultáneamente, un seguimiento a las formas de trabajo que despliegan los estudiantes. Para tal seguimiento se plantearon problemas abiertos y específicos los primeros generales a todos los grupos y contextualizados en los temas que últimamente habían abordado los estudiantes; los especificos tenían que ver con los últimos temas que se habían tocado en el grupo.

Entre los problemas abiertos aparece el de una ranita que está saliendo de un pozo. El enunciado general es: "Cuántos días tarda en salir una ranita que estando en un pozo de 8 metros de profundidad, en el día recorre una distancia ${ }^{\times} 2$ y en la noche resbala una distancia $y$. Siendo 0 Y X 8". En sexto grado $x$ y $y$ fueron valores naturales, en séptimo grado números fraccionarios y en noveno una relación que generaba a $x$ como potencia (en un día anterior recorre el doble del $x$ que recorre el día siguiente).

Los problemas específicos versaban sobre naturales (sexto grado), racionales (séptimo), lógica y potencias (noveno grado), vectores (décimo) y sucesiones (once); tomando la figura 2 se logró identificar cada uno de los grupos a partir de trabajo más generalizado en los estudiantes.

En sexto grado los estudiantes mostraron manejar mucha riqueza en su conocimiento matemático en conexión con el entorno. En el caso de la disciplina predominan elaboraciones sintácticas (pertenecientes a A). Lo anterior se refleja en que en problemas como el de la ranita los estudiantes plantean que la ranita no saldrá del pozo porque se inuere de hambre, revelando gran riqueza en la comprensión de su realidad.

En séptimo grado es más fuerte el trabajo en $\mathrm{A}$ sin conectarlo adecuadamente con 
B. El conocimiento matemático en conexión con el entorno empieza a perder riqueza y empieza a ser de poco interés para los estudiantes. Los elementos intermedios no se manejan como se esperaba, pues si un estudiante sabe sumar dos fracciones se esperaría que con ello proponga un problema, lo que no sucede.

De allí en adelante, en noveno, diez y once sobresalen las preferencias por $\mathrm{A}$ y la ausencia de trabajo con elementos intermedios es total. De otra parte, trabajar en conexión con el entorno deja entrever que ésta se congela o degenera. Por otro lado los tiempos de reflexión alrededor de los problemas decae. Mientras los estudiantes de sexto pueden dedicar media hora a un problema, los de once escasamente dedican cinco minutos. En la medida que aumenta el grado se dedica mayor tiempo a los ejercicios planteados desde $\mathrm{A}$.

Haciendo este estudio y al contrastar los grupos pareciese que las formas de enseñanza y los conocimientos planteados, en vez de acercar a los estudiantes al entorno lo que ha hecho es alejarlos, que olviden lo que en él habían aprendido y dejarlos sin elementos intermedios para lograr tales conexiones. Además a todos ellos es común que lo tratado desde $A$ son aprendizajes poco significativos y retenidos durante poco tiempo. (No es raro ver a estudiantes de noveno preguntando cosas que han visto todos los cuatro cursos anteriores).

\section{¿QUÉ HACER?}

Desde lo planteado anteriormente se deriva una propuesta muy particular: empezar a restablecer las relaciones que se han degenerado hasta casi desaparecer, comenzando por aquellas en las cuales son más fuertes los estudiantes, y respaldando e intentando extender significativamente sus tiempos de reflexión. Por otro lado, seguir orientando la construcción de relaciones que apenas comienzan a tomar forma. Hacer crecer todo lo anterior para irnos acercando, en la medida de lo posible, al esquema correcto.

\section{REFERENCIAS BIBLIOGRÁFICAS}

Eco, Humberto. Tratado de Semiótica General. Segunda edición, Editorial Lumen (Barcelona).

Ramírez, John Freddy. Acercamientos a un modelo democrático en la enseñanza de la fisica, 1997. Trabajo de grado presentado para optar el título de Licenciado en Matemáticas y Física, Universidad del Tolima.

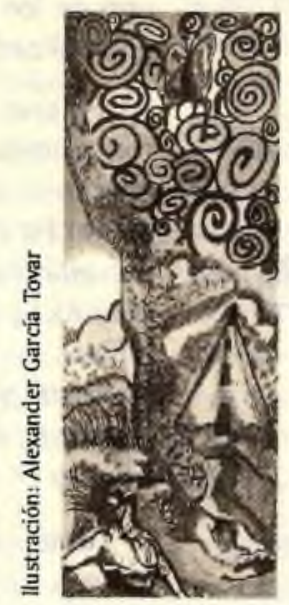

